Journal of

\section{Applied Crystallography}

ISSN 0021-8898

Received 30 May 2000

Accepted 8 August 2000

\title{
Response to Larsen \& Thorkildsen's comments on Extinction-corrected mean thickness and integrated width used in the program UMWEG98
}

\author{
E. Rossmanith \\ Mineralogisch-Petrographisches Institut der Universität Hamburg, D-20146 Hamburg, Grindelallee 48, Germany. \\ Correspondence e-mail: mi2a000@mineralogie.uni-hamburg.de
}

(C) 2000 International Union of Crystallography Printed in Great Britain - all rights reserved
Some additional comments are made concerning the asymptotic expressions for the primary-extinction factor for a perfect spherical crystal.
In Fig. 1 of the paper by Rossmanith (2000), the ratio of the extinction-corrected mean thickness to the extinction length, $t_{\mathrm{ext}} / \Lambda$, of a perfect crystal sphere (solid lines therein) is compared with the results for the semi-infinite plane parallel plate (dotted lines). The asymptotic expressions given by Larsen \& Thorkildsen (1998) for perfect crystal spheres, represented as dashed lines in Fig. 1 of Rossmanith (2000), were questioned by the author.

It is pointed out by Larsen \& Thorkildsen (2000) that for the 'Laue case', the disagreement between their asymptotic expression and the Laue approximation solution is owing to a sign error in their original paper (Larsen \& Thorkildsen, 1998). For large values of the ratio of mean thickness to extinction length, $\bar{t} / \Lambda$, the corrected expression given as equation (1) in the comments by Larsen \& Thorkildsen (2000) now indeed agrees with the solid line 2 in Fig. 1 of Rossmanith (2000), which was derived using the Takagi theory.

For the 'Bragg case', on the other hand, the solid line 1 in Fig. 1 represents a kinematical upper limit for the $t_{\mathrm{ext}} / \Lambda$ ratio of a perfect crystal sphere totally bathed in the incident X-ray beam (the cross section of the incident beam is larger than the cross section of the sample for all sample diameters under consideration!), whereas the dotted curve 3 represents the dynamical solution for the symmetrical Bragg case of a semi-infinite plane parallel plate (the cross section of the incident beam is small compared to the infinite surface of the sample).

According Larsen \& Thorkildsen (2000), equation (2) given in their comments can be used for the calculation of $y_{p}(\theta \rightarrow \pi / 2)$ for a finite convex crystal of general shape bathed in the incident beam. It can easily be shown that by applying equation (2) to a needle-shaped crystal oriented parallel to the incident beam, the result

$$
y_{p}^{\text {needle }}=y_{p}^{\text {plate }}
$$

is obtained, where $y_{p}^{\text {needle }}$ is the extinction factor for the needle bathed in the incident beam and $y_{p}^{\text {plate }}$ is the extinction factor for the semiinfinite plane parallel plate. Having in mind the definition of the extinction factor [Rossmanith, 2000, equation (12) therein], it follows that equation (1) and consequently equation (2) given in the comments of Larsen \& Thorkildsen (2000) are correct only if identical intensity profiles are obtained during the $\omega$ scan for both the needle as well as the semi-infinite plane parallel plate. But, in view of the very different experimental conditions, it seems improbable that the profiles are identical, whatever theory is used, i.e. it should be expected that, because of the well known shape dependence of intensity profiles, they will differ outside the region of total reflection.

Similar arguments hold for all other convex-shaped crystals, which can be considered as made up of needles. As a consequence, neither equation (2) of Larsen \& Thorkildsen (2000) nor the expressions given earlier by Larsen \& Thorkildsen (1998) are exact (analytical) expressions for a perfect spherical crystal in the limit $\theta \rightarrow \pi / 2$.

\section{References}

Larsen, H. B. \& Thorkildsen, G. (1998). Acta Cryst. A54, 511-512. Larsen, H. B. \& Thorkildsen, G. (2000). J. Appl. Cryst. 33, 1447. Rossmanith, E. (2000). J. Appl. Cryst. 33, 330-333. 